\title{
Parent-completed scales for measuring seizure severity and severity of side-effects of antiepileptic drugs in childhood epilepsy: development and psychometric analysis
}

\author{
Hans A. Carpay ${ }^{\text {a, } *}$, Willem F.M. Arts ${ }^{\text {a }}$, Jan Vermeulen ${ }^{\mathrm{b}}$, Hans Stroink ${ }^{\mathrm{c}}$, \\ Oebo F. Brouwer ${ }^{d}$, A.C. Boudewyn Peters ${ }^{\mathrm{e}}$, Cees A. van Donselaar ${ }^{\mathrm{f}}$, \\ Albert P. Aldenkamp ${ }^{\mathrm{b}}$ \\ a Department of Child Neurology, Westeinde Hospital and Juliana Children's Hospital, Den Haag, The Netherlands \\ ' Department of Psychology, 'Meer en Bosch' Epilepsycentre, Heemstede, The Netherlands \\ ' Department of Child Neurology, Sophia Children's Hospital / University Hospital, Rotterdam, The Netherlands \\ 'Department of Child Neurology, University Hospital, Leiden, The Netherlands \\ e Department of Child Neurology, University Hospital and Wilhelmina Children's Hospital, Utrecht, The Netherlands \\ ${ }^{\mathrm{f}}$ St. Clara Hospital, Rotterdam, The Netherlands
}

Received 11 October 1995; revised 2 February 1996; accepted 2 February 1996

\begin{abstract}
We have developed two outcome measures for childhood epilepsy: a seizure severity (SS) scale and a side-effects (SE) scale. Both scales have been designed for completion by parents. The scales were tested in two pilot phases and the results of this stepwise analysis are described here. The final scales' psychometric properties were assessed in a group of 80 children with active epilepsy, representative of the population at whom the scales were aimed: children with chronic epilepsy, aged 4-16 years, including all seizure types and epilepsies, as well as children with neurological comorbidity. The SS scale and SE scale showed good internal consistency and test-retest stability. Although there was a significant positive correlation between the SS scale and the SE scale, this was low, indicating that the scales measure a different clinical trait. The SE scale consisted of two subscales: a Toxic subscale, measuring the severity of dose-related side-effects, and a Chronic subscale, measuring the severity of long-term behavioural and cognitive side-effects. These subscales for side-effects showed a high correlation and can be used as a joint scale. These scales have the potential to improve outcome assessment in childhood epilepsy and they can be used to assess important aspects of quality of life in this population.
\end{abstract}

Keywords: Quality of life: Outcome measure; Seizure severity; Adverse effect; Intractable epilepsy; Children

\footnotetext{
"Corresponding author. Present address: Department of Clinical Neurophysiology, Westeinde Hospital, PO Box 432, 2501 CK Den Haag, The Netherlands. Tel.: + 31 (70) 3302000 ; fax: +31 (70) 3809459 .
}

\section{Introduction}

During the past decade, several tools have been developed to improve the assessment of treatment outcome in epilepsy [19]. These efforts have focused on epilepsy in adults. The high prevalence of epilepsy 
in children [13] and the fact that about $30 \%$ does not achieve total seizure control $[3,12]$, suggest that the development of similar measures of outcome for a childhood epilepsy population is indicated. Such measures can prove useful in research and clinical practice.

The purpose of treating epilepsy is to improve the child's life. It is important to assess the effects of treatment on an individual basis. In the words of Freeman: 'We need to treat seizures when, for that individual, the risk of having seizures and the consequences of having more seizures are worse than the risks and consequences of the treatment itself' [11]. For clinicians concerned with epilepsy, the most important variables are frequency and severity of seizures, and prevalence and severity of side-effects of antiepileptic drugs (AEDs). How can a clinician determine whether an acceptable balance is achieved between seizure suppression and toxicity? In childhood epilepsy, a reliable and valid inventory of the child's health-related quality of life (QoL) [20], would probably provide useful answers $[8,9]$.

QoL is generally seen as a multidimensional concept. In most studies addressing QoL in epilepsy, physical symptoms are neglected compared to psychosocial issues [14]. Measures of seizure severity and severity of side-effects are important aspects of the physical domain of QoL in epilepsy [1]. The methodological difficulties of self-report QoL assessments in a population of children with epilepsy $[9,19]$ suggest that the use of parent-report instead of self-report scales is justified. The majority of children with chronic epilepsy lacks the skills required for reliable self-report, because they are mentally retarded [16] or simply too young.

We describe two newly developed scales for parents of children with epilepsy: the The Hague seizure severity (SS) scale and the The Hague side-effects (SE) scale. These scales were designed to measure the parents' perception of these two basic aspects of severity of epilepsy in children.

\section{Methods}

\subsection{Subjects}

Children were recruited from the outpatient populations of the departments of child neurology of two hospitals in The Hague (one children's hospital and one general hospital) and the University Hospitals of Rotterdam, Leiden and Utrecht, four cities in the most densely populated area of the Netherlands. Parents were asked to participate by their child's own doctor and, after giving informed consent, they were asked to complete a mailed questionnaire, including both scales, at home. All questions referred to the child's condition during the previous 3 months. All parents completed the SS scale. The SE scale was completed only by parents of children who were treated with AEDs.

The treating physicians - experienced child neurologists - classified the seizures and epilepsies according to the current ILAE Classifications [6,7].

\subsubsection{Inclusion criteria}

Children aged 4-16 years, with a diagnosis of epilepsy and having experienced at least one seizure in the past 3 months were included, regardless of seizure type or syndrome diagnosis. The physicians were asked to select parents with sufficient Dutchreading skills to complete a questionnaire, thus, excluding most immigrant parents.

\subsection{Content}

We developed two scales to measure the parents' perception of the severity of seizures (SS scale) and of side-effects of AEDs (SE scale) in children with epilepsy. The 19-item pilot SS scale was a modified version of the Liverpool SS scale - a valid and reliable self-report scale for adults [2] - to which new items were added as suggested by the child neurologists who participated in the study. The definitive 13-item SS scale is presented in Appendix A. Questions 2, 3 and 7-13 have been translated from the Liverpool SS scale and subsequently modified to allow completion by the parents. The Liverpool scale includes some items with precise rather than subjective responses. We felt it was more consistent to include only items with subjective answer categories.

The pilot SE scale was based on items suggested by the child neurologists. It also included an open question inviting the parents to suggest alternative or additional items which they considered important within the scope of the scale. 
Ten sets of parents of children with uncontrolled epilepsy were asked to comment on the items and content of the pilot scales. Some questions were subsequently added or rephrased. To some items in the SS scale and all items in the SE scale, a response category 'unknown' was added, to allow completion in case of a permanent impairment (like mental retardation or cerebral palsy). When a child is always incontinent or unable to express certain complaints, it seems impossible for the parents to answer a question about such problems occurring during or after a seizure, or because of AED treatment. The most severe score possible on both scales for children with (severe) permanent impairments was consequently lower than for children without those impairments.

Next, the scales were tested in two phases: (1) a pilot study in 25 patients, after which a preliminary psychometric evaluation was done; and (2) a subsequent study in a larger population of 80 children and their parents, followed by a more extensive data analysis. We have chosen not to construct these scales on the basis of factor analysis, but first of all to construct the most homogeneous scales possible as suggested by Nunally [17].

\subsection{Psychometric and statistical analyses (SS and SE scales)}

\subsubsection{Item analysis}

Two steps of item analysis were performed. (1) Corrected item-total correlations (CITCs) - the correlation between the item and the rest of the scale were calculated and items with a CITC $<0.20$ were deleted. (2) Frequency distributions of answers were computed and items where one alternative was chosen in $>95 \%$ of cases were left out.

\subsubsection{Reliability analysis}

Crohnbach's alpha was computed as a measure of internal consistency of the final scales. A scale has sufficient internal consistency for research purposes, when alpha is at least: 0.8 [23].

Test-retest reliability: of 22 consecutive parents who were asked to complete a second questionnaire 14 days after the first, 18 parents responded. Pearson's $R$ was used as a measure of test-retest stability.

\subsubsection{Distribution of the scores}

Items consisted of 4 or 5 point adjectival questions. A simple scoring system was adopted with ratings ranging from 1 (most favourable) to 4 or 5 (most unfavourable) points for each item.

Scale mean scores, S.D. values and frequency distributions of scores were established as these measures indicate the scales' potential to measure change.

\subsubsection{Correlations between scales and correlations with seizure frequency}

Spearman's rank correlation coefficient $\left(R_{\mathrm{s}}\right)$ was used as a measure of the relationships between the scales and of each scale with seizure frequency. We used an estimation of seizure frequency in the preceding 3 months by the parents.

\section{Results}

In the final study, 81 children and their parents were included. The parents of one child did not return the questionnaire. The analysis of the SS scale was, thus, based on 80 completed questionnaires. The parents of 75 children completed the SE scale as 5 children were not treated with AEDs. The overall availability of data was excellent. None of the parents had any serious problems completing the scales.

Demographic and clinical characteristics of the children included in the study are shown in Table 1. The majority of the children can be considered to be patients with intractable epilepsy. In $75 \%$ of the children, seizures were not under control after at least 1 year of therapy. Many children had symptomatic epilepsies and seizure types which are difficult to control. Mild to severe mental retardation was present in $37(46 \%)$ children. The distribution of the number of AEDs/child reflected current clinical practice as monotherapy dominated and some children were not treated with an AED despite recurrent seizures. However, as one might expect in this sample, $40 \%$ of the children were treated with polytherapy.

\subsection{SS scale}

\subsubsection{Initial analysis of the pilot study}

One item concerning active seizure control by the child was left out. All 25 parents answered nega- 
Table 1

Demographic and clinical variables of the sample

\begin{tabular}{ll}
\hline Number of children & 80 \\
Mean age & 9.6 years (3.4) \\
Sex & $34(43 \%)$ girls \\
Mean duration of epilepsy & 4.7 years (4.0) \\
Epilepsy classification & Localization-related \\
& - Idiopathic with age-related onset: \\
& $12(15 \%)$ \\
& - Symptomatic: 31 (39\%) \\
& Generalized \\
& - Idiopathic with age-related onset: \\
& $11(14 \%)$ \\
& - Generalized idiopathic or \\
& symptomatic: $18(23 \%)$ \\
& Unclassified: $8(10 \%)$ \\
& Simple partial: 18 \\
& Complex partial: 24 \\
& Secondary generalized: 15 \\
& Absences: 14 \\
& Myoclonic: 3 \\
& Clonic: 1 \\
& Tonic: 3 \\
& Tonic-clonic: 9 \\
& Atonic: 7 \\
No AED: $5(6 \%)$ \\
1 AED: $43(54 \%)$ \\
2 AEDs: $21(26 \%)$ \\
3 AEDs: $10(13 \%)$ \\
4 AEDs: $1(1 \%)$ \\
\end{tabular}

SD values of mean values are given between parentheses.

* Seizure classification relates to seizures in the preceding 3 months; 13 children had $>1$ seizure type.

tively. No other changes were found necessary after the analysis of the pilot results.

\subsubsection{Final SS scale}

After the item analysis, we further reduced the number of items in the SS scale. Items deleted because of a low CITC related to the following symptoms. Interruption of activities by the seizure, disability to speak during the seizure and seizure-related faecal incontinence. For several items relating to the same symptoms, we made a choice based on the best CITC. The final SS scale comprised 13 items (Appendix A.

The items represent the following areas of content. Consciousness (4 questions), motor symptoms (2), incontinence (1), injuries/pain (3) and overall SS (3). Ictal symptoms are addressed in 9 and predominantly postictal symptoms in 4 questions. The
Table 2

Results of reliability analysis of the scales for SS, SE and subscales for toxic side-effects and chronic side-effects

\begin{tabular}{lll}
\hline Scale & Alpha & Retest \\
\hline SS (13 items) & 0.85 & 0.93 \\
SE (20 items) & 0.88 & 0.91 \\
Subscales & & \\
Toxic (14 items) & 0.87 & \\
Chronic (6 items) & 0.81 & \\
\hline
\end{tabular}

Alpha: Crohnbach's alpha (internal consistency). Retest: Pearson's $R$ between first test score and retest score after 14 days.

CITC values are listed in Appendix A. CITC values ranged from 0.22 to 0.70 . The highest CITC, indicating the item most representative of what the scale measures, was found for Q3 (How severe have the seizures been overall?).

Table 2 presents the results of reliability analysis, which indicate good internal consistency (Crohnbach's alpha 0.85 ) and a high test-retest correlation of 0.93 . The distribution of the SS scale scores is
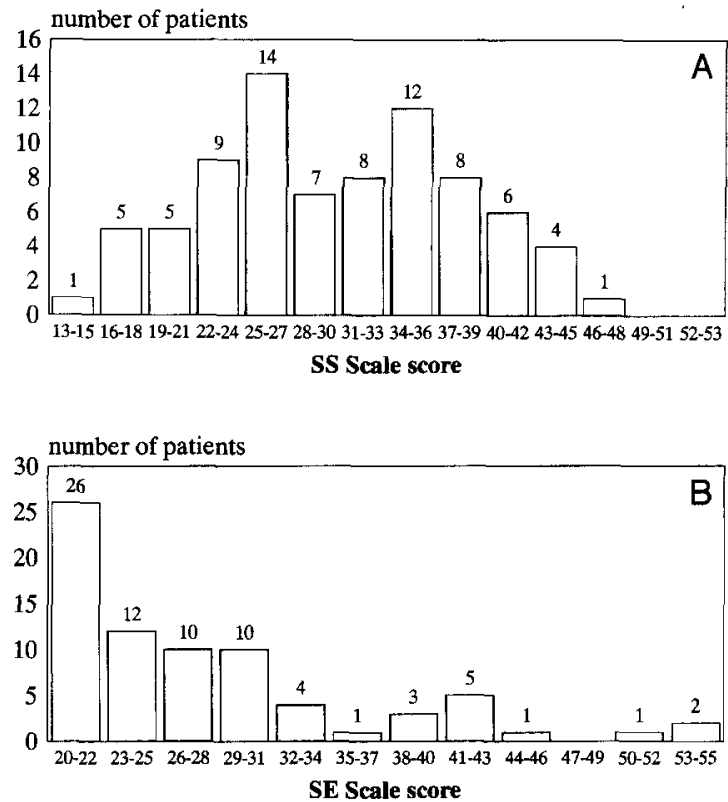

Fig. 1. Frequency distribution of scores. (A) The SS scale: clustered scores in 80 children. Mean score $30.5(8.0)$. Least severe score possible $=13$. Most severe score possible $=54$. (B) The SS scale: clustered scores in 75 children using antiepileptic drugs. Mean score $27.9(8.5)$. Least severe score possible $=20$. Most severe score possible $=80$. SD values of mean values are given between parentheses. 
shown in Fig. 1a. Some scores were obtained on the lower extreme of the scale, which implied that for these children the scale could not measure any improvement. No scores were produced in the upper extreme range of the scale. The mean is $>2$ S.D. values higher than the lowest possible score and 3 S.D. values lower than the highest possible score.

\subsection{SE scale}

\subsubsection{Initial analysis of the pilot study}

The pilot SE scale was revised completely because of ambiguity of certain items and insufficient internal consistency. We frequently found an inconsistency in the parents' response to a question concerning the presence of side-effects $(12 / 24$ parents reported 'no side-effects') and the subsequent responses on a list of: items representing the most common side-effects (10 of these 12 parents responded positively to at least 1 item). Many parents added items to the pilot list of side-effects. Some of these items were included in a new 29 -item version of the SE scale.

\subsubsection{Final SE scale}

After testing the new 29-item version, three items were deleted: one with $100 \%$ negative response (concerning vomiting), two with a low CITC (concerning sleeplessness and increase of appetite/obesity). The final SE scale was divided into three subscales, based on a clinical classification of the sideeffects. The subscales were called 'Toxic' (14 items relating to dose-dependent gastro-intestinal and neurotoxic side-effects), 'Idiosyncratic' (6 items relating to gum hyperplasia rash, hirsutism, hair loss, acne/pimples, itching) and 'Chronic' ( 6 items relating to cognitive and behavioural side-effects). We felt it was appropriate to analyse the internal consistency of these subscales first, before defining the final SE scale.

Internal consistency analysis of the subscales ( $n$ $=75$ ) resulted in the following alpha scores: Toxic 0.87 ; Idiosyncratic 0.47 ; Chronic 0.81. Alpha of the Idiosyncratic subscale was below the limit of 0.8 , this subscale was consequently not included in the subsequent analysis.

Test-retest stability of the SE scale was good (Table 2). Mean scores and score distribution of the
Table 3

Correlations between scores on the scales for SS, toxic side-effects and chronic side effects (Spearman's rank correlation)

\begin{tabular}{llll}
\hline & SE scale & $\begin{array}{l}\text { Toxic } \\
\text { subscale }\end{array}$ & $\begin{array}{l}\text { Chronic } \\
\text { subscale }\end{array}$ \\
\hline SS scale & 0.25 & 0.20 & 0.23 \\
& $P=0.027$ & $P=0.080$ & $P=0.048$ \\
Toxic subscale & & & 0.58 \\
& & & $P<0.001$ \\
\hline
\end{tabular}

$n=75$.

SE scale are shown in Fig. 1b. On the SE scale many children produced a score in the lowest possible range and the mean score was < 1 S.D. from the scale's lowest value. Very few children obtained scores in the high range of the SE scale.

The definitive SE scale is presented in Appendix $B$, including a list of CITC values. The CITC values range from 0.24 to 0.77 . The item with the highest CITC in the Toxic subscale was 'Fatigue' (0.77) and in the Chronic subscale 'Decreased concentration' (0.64).

\subsection{Correlations}

Correlations between the SE scale, Toxic and Chronic SE subscales and the SS scale are shown in Table 3. These indicate that the SS scale and the SE scale measured a different trait as we found a low correlation between them. The Toxic and Chronic subscales largely measured the same clinical trait.

There was a significant correlation between seizure frequency and the score on the SS scale: $R_{\mathrm{s}}$ was $-0.33(P=0.004)$. This means that frequent seizures are less severe. The correlation between seizure frequency and the score on the SE scale was not significant: $R_{\mathrm{s}}$ was $0.18(P=0.12)$.

\section{Discussion}

\subsection{SS scale}

The 13-item SS scale is easy to administer and reliable in terms of internal consistency and retest stability. The distribution of scores obtained in this sample suggest the scale has adequate potential to 
measure both positive and negative change in seizure severity. Its validity cannot be demonstrated using an external gold standard. However, face and content validity were appropriately established. Furthermore, the question with the highest CITC clearly addresses SS, supporting the idea that the scale measures what it was intended to measure. The negative correlation between seizure frequency and the score on the SS scale is in accordance with clinical intuition and suggests that parents are able to separate seizure frequency and severity. Further evidence of construct validity must be obtained from subsequent clinical studies.

It is well-recognized that assessment of SS adds to the overall reliability, sensitivity and clinical relevance of research on treatment outcome in uncontrolled epilepsy $[2,4,5,22,24]$. In addition to a measure of seizure frequency, the SS scale can provide a complete seizure-based outcome system for studies in uncontrolled childhood epilepsy. It is likely that for children with recurrent seizures it provides useful information pertaining to their QoL.

\subsection{SE scale}

The SE scale and its subscales have adequate internal consistency and retest stability. Mean scores were very close to the best possible scores. This indicates that prevalence and severity of side-effects in the majority of the study population were limited. This easily administered scale seems a useful and valid screening tool for identifying children with gastro-intestinal and neurotoxic side-effects. It is important to emphasize that the SE scale was tested in an unselected sample of patients who have been treated for a long time. Tolerance to dose-dependent side-effects may have reduced the scores on side-effects as addressed in the Toxic subscale $[10,15]$. Probably, if children had been selected shortly after starting new medication, higher scores for dose-dependent side-effects might have resulted.

The Idiosyncratic subscale failed the standard of internal consistency. This means that the symptoms and signs grouped in this subscale do not represent a homogeneous construct. The summation of scores over a number of items is appropriate only if all items are measuring the same trait [23]. Although the validity of this subscale seems strong, we can not confidently conclude that a summation of scores on the items addressing idiosyncratic side-effects is appropriate. They may be useful as single items addressing idiosyncratic side-effects. One might argue that the Idiosyncratic subscale's low internal consistency is even supportive of the validity of the individual items.

The considerable correlation between the Toxic and Chronic subscales makes it appropriate to use one SE scale including the items of both subscales, instead of separate subscales. We found no significant correlation between seizure frequency and the score on the SE scale. This suggests that parents are able to separate seizures from side-effects. However, the complex relations between seizures and side-effects clearly warrant further studies.

A quantification of subjective adverse effects is of importance in epilepsy treatment [19]. Using a scale listing the most frequent side-effects may be a particularly informative approach. In our pilot study, the majority of parents, reporting 'no side-effects' to a global question, did report complaints on a subsequently presented list of side-effects. As such, the SE scale may contribute to a better screening for the presence of side-effects as well as to the assessment of their severity.

\subsection{Implications for future research and applica- tions}

Health measurement scales are time-consuming instruments. Consequently, focusing on relevant dimensions might increase their acceptability. It is probable that for children with uncontrolled seizures, SS and severity of side-effects of AEDs are relevant issues.

For an assessment of SS and severity of side-effects in children, population-specific scales are indicated as scales developed for an adult population are not appropriate [19]. As self-report in young children is not a reliable alternative, it seems justified to apply parent-report instruments for QoL assessments in childhood disease [18]. This would also seem to be a sensible approach in retarded children or adolescents, when they are still being cared for by their parents. Some considerations favour the use of parents rather than physicians as the source of information about seizures and side-effects. (1) Seizures are 
rarely witnessed by the physician. Any assessment of their severity by the physician will be based on a description by others and by general medical perceptions. (2) The severity of most side-effects can not be assessed objectively. Cognitive and behavioural side-effects may be examined with standardized neuropsychological tests, but these are not as easily administered as the SE scale, and not always appropriate for this population. It should be noted that patient - or parent - perceived severity of disease does not necessarily parallel the physician's view [21]. We are conducting a study to compare the parents' scores on the SS and SE scales and the physicians' clinical global impression of SS and severity of side-effects.

Sensitivity to clinically relevant change is an important quality of any clinimetric instrument developed to assess treatment effects. We are investigating this issue in a 1-year follow-up study using the SS scale and SE scale. Further studies in a larger sample are conducted to provide more data on the clinical validity of both scales. An English version of the Dutch SS and SE scales has been completed and will be subjected to a similar study to assess their reliability and validity in a comparable Western inner city population.

\section{Acknowledgements}

These investigations were supported by the Research Committee on Epilepsy of the National Epilepsy Fund (Project CLEO A108). The authors are indebted to Drs. C.E. Catsman-Berrevoets, L.A.E.M. Laan en O. van Nieuwenhuizen for providing patients. Dr. S. Shinnar (Montefiore Medical Center, New York, USA) is gratefully acknowledged for reviewing the English version of the scales.

\section{Appendix A. SS scale and CITC values in 80 children}

Questions relate to your child's seizures in the past 3 months.

Q1. How often do you notice a decrease of consciousness during a seizure in your child? $($ CITC $=$ 0.33) a. always

b. usually

c. sometimes

d. never

Q2. How long does such a decrease of consciousness last? (From time of onset to time of normal consciousness) $(C I T C=0.59)$
a. very long
b. long
c. short
d. very short

Q3. How severe have the seizures been overall? $(C I T C=0.70)$
a. very severe
b. severe
c. mild
d. very mild

Q4. Are there any muscle jerks or cramps in the arms or legs during an attack? $(C I T C=0.48)$
a. always
b. usually
c. sometimes
d. never

Q5. How long do the jerks or cramps last during an attack? $(C I T C=0.54)$
a. very long
b. long
c. short
d. very short

e. does not apply, there are no jerks or cramps

Q6. How noticeable are the seizure symptoms? $($ CITC $=0.64)$

a. very noticeable, everyone will notice an attack

b. fairly noticeable, most people will notice an attack

c. not very noticeable, most people will not notice

d. not at all noticeable, you have to be very alert to notice an attack

Q7. During or after an attack, how often does your child seem confused? $(C I T C=0.49)$
a. always
b. usually
c. sometimes
d. never

Q8. During an attack, how often does your child wet him/herself? $($ CITC $=0.41)$
a. always
b. usually 
c. sometimes

d. never or unknown, my child is permanently incontinent

Q9. During an attack, how often does your child bite his/her tongue? (CITC 0.49)
a. always
b. usually
c. sometimes
d. never

Q10. How often does your child become injured during an attack (other than biting the tongue)? $(C I T C=0.22)$
a. always
b. usually
c. sometimes
d. never

Q11. After the attack has finished, is your child sleepy? (including sleepiness caused by the use of rescue medication like diazepam) $(C I T C=0.67)$
a. always
b. usually
c. sometimes
d. never

Q12. After an attack, does your child complain of sickness, headache and/or pain in the muscles? $(C I T C=0.43)$
a. always
b. usually
c. sometimes to complain about that
a. very long
b. long
c. short
d. very short or direct after an attack

d. never or unknown, my child would not be able

Q13. After an attack, how long does it take, until your child can resume normal activity? $($ CITC $=0.65)$

\section{Appendix B. The SE scale and CITC values in 75 children}

The following questions concern possible side-effects of the medication for epilepsy. To explain: If your child has trouble walking, and you believe that this is caused by the medication, this is called a side-effect. If this difficulty with walking has another (probable) cause, e.g. a handicap or a broken leg, this does not count as a side-effect.

Have you noticed any of the following side-effects of antiepileptic medication in your child during the past 3 months?

$\mathrm{a}=\mathrm{yes}$, it is a very serious problem

$\mathrm{b}=$ yes, it is a moderately serious problem

$c=$ yes, it is a mild problem

$\mathrm{d}=$ no or not applicable or cannot be assessed because of impairment

\section{CITC}

1 drowsiness, sleepiness a. b. c. d. 0.62

2 dizziness a. b. c. d. 0.48

3 uncertainty when walking a. b. c. d. 0.66

4 falling

5 sickness

6 difficulty with defecation

7 diarrhoea

8 shaking, trembling

9 speech difficulties

10 double or blurred vision

11 headache

12 fatigue

13 loss of appetite

14 depression

a. b. c. d. 0.56

a. b. c. d. 0.43

a. b. c. d. 0.31

a. b. c. d. 0.24

a. b. c. d. 0.45

a. b. c. d. 0.41

a. b. c. d. 0.49

a. b. c. d. 0.55

a. b. c. d. 0.77

a. b. c. d. 0.40

a. b. c. d. 0.54

a. b. c. d. 0.31

15 hyperactivity

a. b. c. d. 0.32

a. b. c. d. 0.61

a. b. c. d. 0.63

a. b. c. d. 0.64

a. b. c. d. 0.57

19 decreased concentration

20 behavioural disturbance

CITCs relate to the full SE scale, which comprises a subscale for toxic side-effects (items 1-14), and for chronic side-effects (items 15-20).

\section{References}

[1] Baker, G.A., Health-related quality-of-life issues: optimizing patient outcomes, Neurology, 45 (Suppl. 2) (1995) S29-S34.

[2] Baker, G.A., Smith, D.F., Dewey, M., Morrow, J., Crawford, P.M. and Chadwick, D.W., The development of a seizure severity scale as an outcome measure in epilepsy, Epilepsy Res., 8 (1991) 245-251.

[3] Berg, A.T., Hauser, W.A. and Shinnar, S., The prognosis of childhood-onset epilepsy. In: S. Shinnar, N. Amir and D. Branski (Eds.), Childhood Seizures. Pediatric and Adolescent Medicine. Vol 6, Karger, Basel, Switzerland, 1995, pp. 9399. 
[4] Blume, W.T., Uncontrolled epilepsy in children, Epilepsy Res., 9 (Suppl. 5) (1992) 19-24.

[5] Commission on antiepileptic drugs of the ILAE, Guidelines for antiepileptic drug trials in children, Epilepsia, 35 (1994) 94-100.

[6] Commission on Classification and Terminology of the ILAE, Proposal for revised clinical and electroencephalographic classification of epileptic seizures, Epilepsia, 22 (1981) 489501.

[7] Commission on Classification and Terminology of the ILAE, Proposal for revised classification of epilepsies and epileptic syndromes, Epilepsia, 30 (1989) 389-399.

[8] Devinsky, O., Outcome research in neurology: incorporating health-related quality of life, Ann. Neurol., 37 (1995) 141142.

[9] Dodson, W.E., Quality of life measurements in children with epilepsy. In: M.R. Trimble and W.E. Dodson (Eds.), Epilepsy and quality of life, Ràven, New York, NY, 1994, pp. 217226.

[10] Engel, J., Seizures and epilepsy, F.A. Davis Company, Philadelphia, 1989, pp. 380-409.

[11] Freeman, J.M., A clinical approach to the child with seizures and epilepsy, Epilepsia, 28 (Suppl. 1) (1987) S103-S109.

[12] Hauser, W.A., The natural history of drug resistant epilepsy: epidemiologic considerations, Epilepsy Res. (Suppl. 5) (1992) 25-28.

[13] Hauser, W.A., The prevalence and incidence of convulsive disorders in children, Epilepsia, 35 (Suppl. 2) (1994) S1-S6.

[14] Hermann, B.P., Quality of life in epilepsy, J. Epilepsy, 5 (1992) 153-165.

[15] Homan, R.W., Miller, B. and Veterans Administration Epilepsy Cooperative Study Group, Causes of treatment failure with antiepileptic drugs vary over time, Neurology, 37 (1987) 1620-1623.
[16] Huttenlocher, P.R. and Hapke, R.J., A follow-up study of intractable seizures in childhood, Ann. Neurol., 28 (1990) 699-705.

[17] Nunally, J.C., Psychometric Theory, McGraw Hill, New York, NY, 1967, pp. 254-258.

[18] Rosenbaum, P., Cadman, D. and Kirpalani, H., Pediatrics: assessing quality of life. In: B. Spilker (Ed.), Quality of Life Assessments in Clinical Trials, Raven, New York, NY, 1990, pp. 205-215.

[19] Carpay, J.A. and Arts, W.F.M., Outcome assessment in epilepsy: a review of available rating scales for adults and methodological issues pertaining to the development of scales for childhood epilepsy. Epilepsy Res., 24 (1996) 127-136.

[20] Schipper, H., Clinch, J. and Powell, V., Definitions and conceptual issues. In: B. Spilker (Ed.), Quality of Life Assessments in Clinical Trials, Raven, New York, NY, 1990, pp. 11-25.

[21] Slevin, M.L., Plant, H., Lynch, D., Drinkwater, J. and Gregory, W.M., Who should measure quality of life, the doctor or the patient? Br. J. Cancer, 57 (1988) 109-112.

[22] Smith, D.F., Baker, G.A., Dewey, M., Jacoby, A. and Chadwick, D.W., Seizure frequency, patient-perceived seizure severity and the psychosocial consequences of intractable epilepsy, Epilepsy Res., 9 (1991) 231-241.

[23] Streiner, D.L. and Norman, G.R., Health Measurement Scales. A Practical Guide to Their Development and Use, Oxford Medical Publications, Oxford, UK, 1989.

[24] Vickrey, B.G., Hays, R.D., Engel, J., et al., Outcome assessment for epilepsy surgery: the impact of measuring health-related quality of life, Ann. Neurol, 37 (1995) 158-166. 